\title{
Zuchtleistung stoffwechselgesunder und diabetischer Chinesischer Streifenhamster (Cricetulus griseus)
}

\author{
O. Loge und H. SteinBeck
}

Hauptlaboratorium der Schering AG, Berlin

Received: December 13, 1968

\begin{abstract}
Breeding Performance of Nornal and Diabetic Chinese Hamsters (Oricetulus griseus)

Summary. The breeding performance of healthy and hereditary diabetic Chinese hamsters was compared. Litter size and litter sequence had no influence on birth weight and growth until weaning. No difference between the body weights of the offspring of healthy and diabetic mothers was found, whereas the mortality rate was higher in the offspring of diabetic mothers. With advancing generation succession there was a tendency to earlier manifestation of diabetes.
\end{abstract}

\section{Elevage de hamsters chinois sains et diabétiques}

Résumé. Les caractéristiques des élevages de hamsters chinois sains et de hamsters héréditairement diabétiques ont été comparées. Le nombre de petits dans la portée et l'ordre de celle-ci n'ont aucune influence sur le poids à la naissance et sur la croissance jusqu'au sevrage. On n'a trouvé aucune différence entre les poids corporels des descendants de mères normales et de mères diabétiques, cependant le taux de mortalité était plus élevé chez les descendants de mères diabétiques. Au cours des générations successives il apparaissait une tendance à la manifestation plus précoce du diabète.

Zusammenfassung. Die Zuchtleistungen stoffwechselgesunder und erblich diabetischer Chinesischer Streifenhamster wurden verglichen. Wurfgröße und Wurffolge hatten keinen Einfluß auf das Geburtsgewicht und das Wachstum bis zum Absetzen. Zwischen Nachkommen stoffwechselgesunder und diabetischer Muttertiere bestand Kein Unterschied hinsichtlich der Körpergewichte, die Mortalitätsrate war jedoch höher bei Nachkommen diabetischer Mütter. Mit steigender Generationsfolge zeigte sich eine Tendenz zu früherer Diabetes-Manifestation.

Key-words: Zuchtleistung, Diabetesmanifestationsalter. Ketonurie, Mortalität, Implantationsrate.
Der Streifenhamster hat wegen des Auftretens eines erblichen spontanen Diabetes mellitus Bedeutung für die Diabetesforschung erlangt (Meier und Yerganian 1959, Meier 1961, Meier und Yerganian 1961, Butler 1967). Eine Besonderheit ist bei diesen Tieren das Fehlen einer Adipositas, die bei den meisten übrigen bekannten Laboratoriumstieren mit hereditärem Diabetes mit dem Manifestwerden der Krankheit korreliert ist.

Seit 1966 werden in unserem Hauptlaboratorium Chinesische Streifenhamster gezïchtet.

Über die Zucht und Haltung des Streifenhamsters wurde zwar schon von anderen Autoren berichtet (Hsieh 1919, Chang und Wu 1938, Herter und Rauch 1956, Smith 1957, Yerganian 1958, Moore 1965, Yerganian 1966, über den Einfluß von Wurfgröße und Wurffolge auf das Geburtsgewicht und die weitere Entwicklung der Jungtiere bis zum Absetzen liegen unseres Wissens in der Literatur keine Angaben vor. Anhand dieser Daten wird erstmalig der Versuch unternommen, die Zuchtleistungen stoffwechselgesunder und diabetischer Muttertiere zu vergleichen, um eventuelle Einflüsse der diabetischen Stoffwechsellage der Mütter auf das Geburtsgewicht festzustellen.

Neben Daten über die Zuchtleistung gesunder und diabetischer Muttertiere werden in der vorliegenden Arbeit auch Beobachtungen über das Alter bei Auftreten von Diabetes in Abhängigkeit von der Generationsfolge und die Häufigkeit einer Ketose mitgeteilt.

\section{Material und Methoden}

Die Hamster werden in Makrolon ${ }^{\circledR}$-Käfigen Typ III auf Hobelspänen bei einer Raumtemperatur von $22-$ $24^{\circ} \mathrm{C}$ und einer Luftfeuchtigkeit von $50-60 \%$ unter konventionellen Bedingungen gehalten. Tageslicht hat zu den Zuchträumen ungehindert Zugang, so daß die Beleuchtungsdauer und -intensität jahreszeitlichen und wetterbedingten Schwankungen unterworfen ist. Im Winter werden die Räume von 6.00 bis $18.00 \mathrm{Uhr}$ künstlich beleuchtet. Die Diät besteht aus einem Gemisch von Hafer, Haferflocken und Sonnenblumenkernen zu gleichen Teilen, Latz ${ }^{\circledR}$ Standardkost, zweimal wöchentlich Salat und Mohrrüben, Wasser ad $l i$ bitum.

Die Zucht wurde 1966 mit 5 männlichen und 8 weiblichen Tieren begonnen ${ }^{1}$ ) und von der 1. Filialgeneration $a b$ ausschließlich durch Bruder-SchwesterPaarung vermehrt. Wurfgeschwister blieben zur Zuchtreife beisammen, sichtbar tragende Weibchen wurden separiert und nach Absetzen ihrer 21 Tage alten Jungen zu den Geschwistern zurückgesetzt. Als diabetisch wurden Tiere bezeichnet, die bei der Testung mit Glucoteststreifen eine positive Harnzucker. reaktion zeigten. Das Vorhandensein einer Ketose wurde bei Feststellung von Ketonkörpern (Aceton,

1 Diese Tiere wurden von der Children's Cancer Research Foundation, Boston, bezogen. Daten über die genetischen Merkmale (z.B. Diabetes-Häufigkeit) der Ursprungskolonie waren nicht erhältlich. 
Acetessigsäure) im Harm durch Ketostix ${ }^{\circledR}$ angenommen.

Der beim Anfassen von den Hamstern spontan abgegebene Harn wurde direkt auf die Teststreifen gebracht, wie von Gerritsen und Dulin (1967) beschrieben.

Die Untersuchungen hinsichtlich der Wurfgröße, des Gewichtes der Jungen bei der Geburt, der Gewichtszunahme bis zum 21. Lebenstage, des Einflusses der Anzahl Junger pro Wurf auf die Entwicklung bis zum Absetzen und hinsichtlich der Zuchtleistung der Hamster über 4 Würfe wurden an 244 Würfen nichtdiabetischer Hamster mit 1519 Jungen durchgeführt.

Für die Beurteilung der Zuchtleistung diabetischer Hamster standen 83 erste Würfe mit 361 Jungen zur Verfügung. Bisher war es nur in wenigen Fällen möglich, mehrere Würfe eines nichtbehandelten diabetischen Hamsters zu beobachten.

Für den Vergleich der mittleren Tiereinzelgewichte von Nachkommen aus Würfen mit unterschiedlicher Anzahl Junger wurde der Scheffé-Test herangezogen (Weber 1967).

\section{Ergebnisse}

Bei den Nachkommen nichtdiabetischer Hamster wurden am 1., 7., 14. und 21. Tag nach der Geburt keine signifikanten Unterschiede (Scheffé-Test) zwischen den Gewichten der Nachkommen aus Würfen mit 3 bis 9 Jungen pro Wurf gefunden. Dies trifft fiür alle Würfe zu (Tabelle 1). Die Regressionsgeraden für das Wurfgewicht in Abhängigkeit von der Anzahl Junger je Wurf für erste bis vierte Würfe haben annähernd den gleichen Verlauf. Mit Hilfe der Varianzanalyse wurden zwar signifikante Unterschiede der Einzeltiergewichte zwischen den Würfen (1. bis 4. Wurf) und auch zwischen den Anzahlen Junger je Wurf gefunden. Diese Unterschiede gehen aber nicht über $10 \%$ hinaus und dürften damit bedeutungslos sein. Wir haben daher aus allen Würfen das durchschnittliche Geburtsgewicht ermittelt, es beträgt $1.68 \mathrm{~g}$ (Spannweite der Mittelwerte 1.59 bis $1.94 \mathrm{~g}$ ). Das mittlere Gewicht beim Absetzen am 21. Tag beträgt $14.04 \mathrm{~g}$ (Spannweite der Mittelwerte 13.65 bis $16.07 \mathrm{~g}$ ).

Der Wurfabstand $(\bar{x} \pm s \bar{x})$ beträgt zwischen

1. und 2. Wurf $48.5 \pm 1.95$ Tage, $n=90$

2. und 3. Wurf $46.7 \pm 1.36$ Tage, $n=77$

3. und 4. Wurf $48.2 \pm 2.27$ Tage, $n=55$

Zwischen den Wurfabständen bestehen keine signifikanten Unterschiede ( $t$-Test).

Das mittlere Geburtsgewicht der Nachkommen diabetischer Hamster beträgt $1.75 \mathrm{~g}$ (Spannweite der Mittelwerte 1.67 bis $1.85 \mathrm{~g}$ ), das Gewicht am 21.Tag post partum $13.86 \mathrm{~g}$ (Spannweite der Mittelwerte 13.20 bis $14.67 \mathrm{~g})$. $Z_{\text {wischen }}$ den errechneten mittleren Tiereinzelgewichten von Jungen aus Würfen mit 3 bis 7 Tieren bestehen keine signifikanten Unterschiede am 1. Lebenstag. Auch am 7., 14. und 21. Tag konnten für
Würfe mit 3 bis 7 Jungen keine Unterschiede nachgewiesen werden.

Ein Vergleich der mittleren Tiereinzelgewichte von Nachkommen nichtdiabetischer und diabetischer Muttertiere (1. Würfe) ergab signifikante Gewichtsunterschiede nur für Tiere aus Würfen mit 5 Jungen am 14. und 21. Tag. Die Nachkommen diabetischer Mütter waren am 14. Tag $1.16 \mathrm{~g}$ und am 21 . Tag $1.88 \mathrm{~g}$ leichter als die Jungen nichtdiabetischer Muttertiere (vgl. Tabelle 1 und 2).

Diabetische Hamster hatten häufiger Würfe mit geringer Anzahl Junger je Wurf als nichtdiabetische Hamster (Abb. 1). Der Unterschied ist hochsignifikant (Chiquadrat-Test). Eine Zählung der Implantate am 8. Tag der Trächtigkeit (Spermiennachweis $=$ Tag 1) bei 20 stoffwechselgesunden und 20 diabetischen Hamstern brachte das gleiche Ergebnis. Diabetische Hamster hatten weniger Implantate als normale Hamster. Der Unterschied konnte statistisch gesichert werden (t-Test).

Die Ausfälle bei 1. Würfen betragen bis zum 21 . Lebenstag $16.3 \%$ bei Jungen nichtdiabetischer und $48.7 \%$ bei Jungen diabetischer Muttertiere. Die Sterblichkeit war in beiden Gruppen zwischen dem 1. und 7. Lebenstag am gröBten (14.1\%:44.9\% (Tabelle 3).

Die Ausfälle bei Nachkommen stoffwechselgesunder Hamster betrugen über 4 Würfe $11.6 \%$. Die Mortalität war bei 1 . Würfen am höchsten.

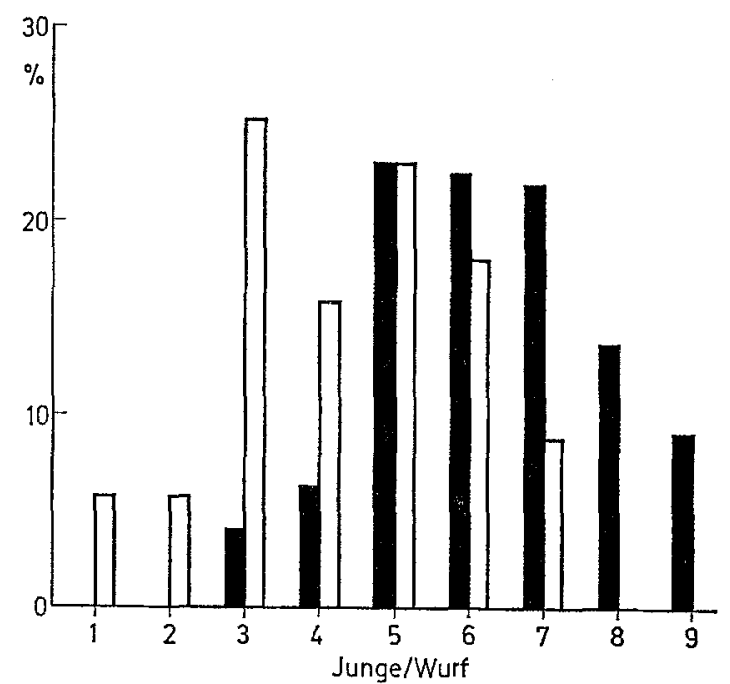

Abb. 1. Prozentuale Häufigkeit von Würfen mit 1 bis 9 Jungen bei diabetischen (helle Säulen) und nicht-diabeti. schen Chinesischen Streifenhamstern (dunkle Säulen)

Seit Bestehen der Kolonie wurden insgesamt 5378 Hamster auf Harnzuckerausscheidung geprüft. Davon waren $52.4 \%$ männlichen und $\mathbf{4 7 . 6} \%$ weiblichen $\mathrm{Ge}-$ schlechts. 1174 Tiere, $42.4 \%$ männliohe und $57.6 \%$ weibliche, konnten als diabetisch bezeichnet werden.

Die Verteilung der Häufigkeit des Diabetes mellitus auf die einzelnen Generationen und die Beteiligung der Geschlechter ist in Tabelle 5 zusammengefaßt. 
Durch Untersuchungen an 490 Hamstern wurde versucht, zu klären, bei welchem Alter der Tiere sich der Diabetes mellitus manifestiert. Es wurden Hamster berücksichtigt, die zwischen dem 60 . und 80 . Lebenstag erstmalig und dann im Abstand von 4 Wochen auf Harnzuckerausscheidung getestet worden sind (Abb. 2).
Die Männchen waren an dieser Erkrankung mit 52.2\% und die Weibchen mit $\mathbf{4 7 . 8 \%}$ beteiligt.

\section{Diskussion}

Die Geburtsgewichte der Streifenhamster sind unabhängig von der Wurfgröße und der Wurffolge konstant. Zwischen den Einzeltiergewichten aus großen

Tabelle 1. Aufzuchtleistung nichtdiabetischer Muttertiere

\begin{tabular}{|c|c|c|c|c|c|}
\hline $\begin{array}{l}\text { 1. Würfe } \\
\text { Anzahl } \\
\text { Junge } \\
\text { pro Wurf }\end{array}$ & $\begin{array}{l}\text { Anzahl } \\
\text { der } \\
\text { Würfe }\end{array}$ & $\begin{array}{l}\text { Mittleres T } \\
\text { 1. Tag }\end{array}$ & $\begin{array}{l}\text { inzelgewicht } \\
\text { 7. Tag }\end{array}$ & $\begin{array}{l}\text { in Gramm } \\
\text { 14. Tag }\end{array}$ & 21. Tag \\
\hline $\begin{array}{l}3 \\
4 \\
5 \\
6 \\
7 \\
8 \\
9\end{array}$ & $\begin{array}{r}4 \\
5 \\
30 \\
26 \\
17 \\
7 \\
5\end{array}$ & $\begin{array}{l}1.70 \pm 0.22 \\
1.71 \pm 0.10 \\
1.75 \pm 0.17 \\
1.70 \pm 0.17 \\
1.68 \pm 0.12 \\
1.59 \pm 0.15 \\
1.61 \pm 0.08\end{array}$ & $\begin{array}{l}3.12 \pm 1.81 \\
5.37 \pm 0.51 \\
5.49 \pm 0.84 \\
5.17 \pm 0.55 \\
5.17 \pm 0.46 \\
4.92 \pm 0.34 \\
4.83 \pm 0.28\end{array}$ & $\begin{array}{r}10.57 \pm 0.96 \\
9.94 \pm 1.15 \\
10.12 \pm 1.43 \\
9.57 \pm 1.06 \\
9.51 \pm 1.06 \\
9.73 \pm 1.14 \\
8.83 \pm 0.41\end{array}$ & $\begin{array}{l}16.07 \pm 0.51 \\
14.82 \pm 1.30 \\
15.08 \pm 1.58 \\
14.58 \pm 0.99 \\
14.41 \pm 1.17 \\
14.69 \pm 1.15 \\
13.80 \pm 0.59\end{array}$ \\
\hline $\begin{array}{l}\text { 2. Würfe } \\
3 \\
4 \\
5 \\
6 \\
7 \\
8 \\
9\end{array}$ & $\begin{array}{r}3 \\
2 \\
11 \\
10 \\
15 \\
10 \\
10\end{array}$ & $\begin{array}{l}1.69 \pm 0.42 \\
1.94 \pm 0.08 \\
1.84 \pm 0.23 \\
1.87 \pm 0.22 \\
1.74 \pm 0.18 \\
1.66 \pm 0.10 \\
1.65 \pm 0.23\end{array}$ & $\begin{array}{l}6.10 \pm 1.46 \\
4.72 \pm 0.33 \\
5.22 \pm 0.98 \\
5.51 \pm 0.55 \\
5.43 \pm 0.68 \\
4.77 \pm 0.38 \\
4.84 \pm 0.51\end{array}$ & $\begin{array}{r}11.35 \pm 4.36 \\
7.02 \pm 0.73 \\
10.20 \pm 2.17 \\
9.19 \pm 1.62 \\
10.14 \pm 1.37 \\
9.31 \pm 0.94 \\
9.34 \pm 1.04\end{array}$ & $\begin{array}{l}15.40 \pm 2.65 \\
14.06 \pm 2.11 \\
15.24 \pm 1.84 \\
13.78 \pm 1.93 \\
14.68 \pm 1.38 \\
14.46 \pm 0.58 \\
13.90 \pm 1.12\end{array}$ \\
\hline $\begin{array}{l}\text { 3. Würfe } \\
4 \\
5 \\
6 \\
7 \\
8 \\
9\end{array}$ & $\begin{array}{r}5 \\
8 \\
11 \\
13 \\
11 \\
4\end{array}$ & $\begin{array}{l}1.79 \pm 0.25 \\
1.88 \pm 0.15 \\
1.85 \pm 0.20 \\
1.81 \pm 0.24 \\
1.66 \pm 0.16 \\
1.76 \pm 0.21\end{array}$ & $\begin{array}{l}5.65 \pm 1.51 \\
5.67 \pm 0.75 \\
5.41 \pm 0.52 \\
5.49 \pm 0.68 \\
5.02 \pm 0.64 \\
5.14 \pm 0.67\end{array}$ & $\begin{array}{r}9.85 \pm 1.62 \\
10.66 \pm 1.40 \\
10.31 \pm 0.90 \\
9.67 \pm 1.60 \\
9.60 \pm 1.82 \\
8.76 \pm 1.72\end{array}$ & $\begin{array}{l}15.15 \pm 1,99 \\
15.23 \pm 1.10 \\
14.58 \pm 0.77 \\
14.88 \pm 2.83 \\
14.38 \pm 2.35 \\
13.66 \pm 1.03\end{array}$ \\
\hline $\begin{array}{l}\text { 4. Würfe } \\
\mathbf{3} \\
4 \\
5 \\
6 \\
7 \\
8 \\
9\end{array}$ & $\begin{array}{l}\mathbf{3} \\
\mathbf{3} \\
\mathbf{7} \\
8 \\
8 \\
\mathbf{5} \\
\mathbf{3}\end{array}$ & $\begin{array}{l}1.70 \pm 0.17 \\
1.84 \pm 0.36 \\
1.77 \pm 0.18 \\
1.72 \pm 0.14 \\
1.84 \pm 0.19 \\
1.66 \pm 0.16 \\
1.72 \pm 0.20\end{array}$ & $\begin{array}{l}4.97 \pm 0.70 \\
5.81 \pm 1.26 \\
5.20 \pm 0.46 \\
5.60 \pm 0.44 \\
5.37 \pm 0.73 \\
5.25 \pm 0.67 \\
5.44 \pm 1.31\end{array}$ & $\begin{array}{r}9.80 \pm 2.11 \\
11.00 \pm 2.36 \\
9.12 \pm 1.07 \\
9.46 \pm 1.01 \\
9.95 \pm 1.43 \\
9.22 \pm 1.84 \\
9.47 \pm 0.21\end{array}$ & $\begin{array}{l}15.15 \pm 2.08 \\
14.82 \pm 1.59 \\
14.21 \pm 1.61 \\
13.65 \pm 0.70 \\
14.27 \pm 1.00 \\
13.71 \pm 1.30 \\
14.58 \pm 1.20\end{array}$ \\
\hline
\end{tabular}

Tabelle 2. Aufzuchtleistung diabetischer Muttertiere

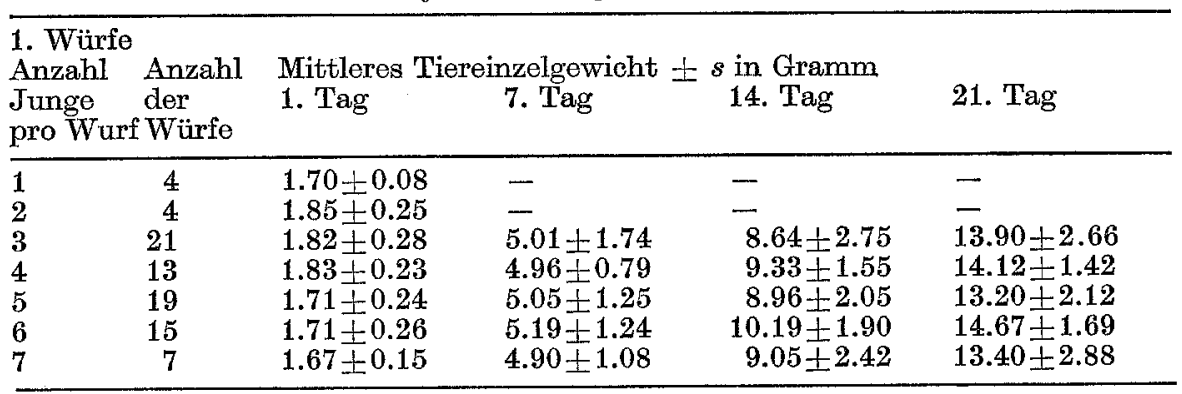

Es ist die Tendenz erkennbar, daß sich der Diabetes in höheren Inzuchtgenerationen früher manifestiert.

Auf das Vorhandensein von Ketonkörpern im Harn wurden 551 Hamster untersucht. Aus Tabelle 4 ist die Verteilung auf die Generationen 3 bis 7 und die Beteiligung der Geschlechter ersichtlich. Insgesamt hatten $74.4 \%$ der diabetischen Hamster eine Ketose. und aus kleinen Würfen besteht kein Unterschied. Dies gilt sowohl für Nachkommen gesunder Hamster als auch für Jungtiere von diabetischen Müttern. Das heißt, daß es für das Geburtsgewicht des Hamsters bedeutungslos ist, ob 3 oder 9 Junge ausgetragen werden. Dieser ungewöhnliche Befund steht im Gegensatz zu der allgemein bekannten Tatsache, daß bei mehrge- 


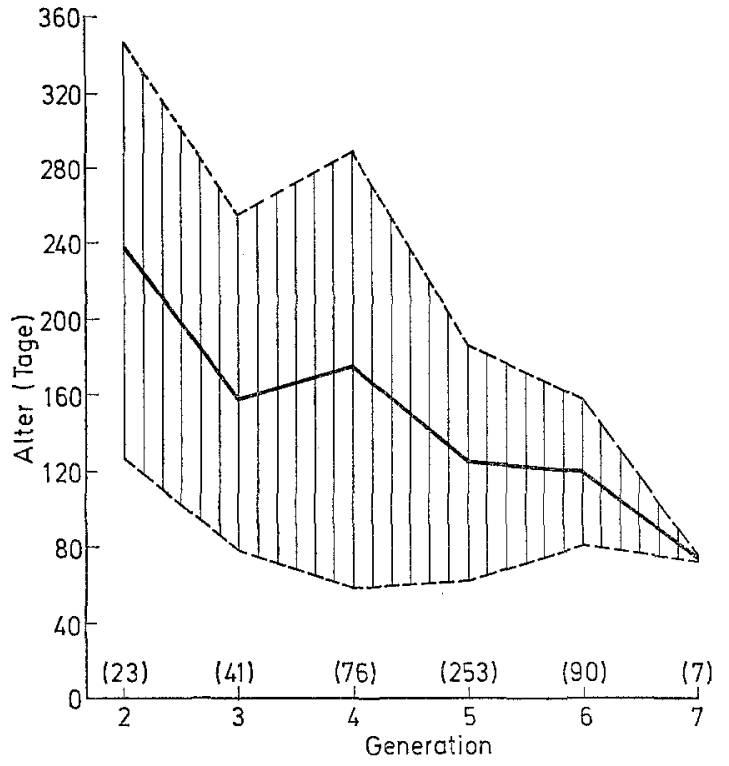

Abb. 2. Alter Chinesischer Streifenhamster bei Entdeckung der ersten Harnzuckerausscheidung (Mittelwert \pm s) bärenden Tieren die Geburtsgewichte mit steigender Wurfgröße abnehmen, wie zum Teil sehr umfangreiche statistische Untersuchungen an Ratten (King 1935), Kaninchen (Wishart und Hammond 1933, Hammond 1934), Meerschweinchen (Ibsen 1928) und Schweinen (Omtvedt et al. 1966, Opicha und Opicha 1944) zeigten. Auch während der Säugezeit ist das Wachstum der Streifenhamster sehr einheitlich, zwischen den mittleren Einzeltiergewichten am 7., 14. und 21. Tag post partum wurden keine signifikanten Unterschiede gefunden.

Die Milchleistung des Hamsters reicht offenbar zur optimalen Aufzucht auch von Würfen mit 9 Jungen aus. Yerganian (1958) berichtet, daß von ihm als Ammen benutzte Hamsterweibchen bis zu 16 Junge aufgezogen haben. Im Gegensatz dazu fanden Steinbeck und $\nabla$. Berswordt (1966) bei jungen Ratten aus Würfen mit 4 und 12 Jungen am 14. Lebenstag durchschnittliche Gewichte von 35 bzw. $23 \mathrm{~g}$. Zu ähnlichen Resultaten kamen Smith und Donald (1937) bei Schweinen. Sowohl bei Ratten als auch bei Schweinen besteht eine positive Korrelation zwischen Wurfgröße und Milch-

Tabelle 3. Aufzuchtverluste bis zum 21. Lebenstag bei Jungen nichtdiabetischer und diabetischer Muttertiere

\begin{tabular}{|c|c|c|c|c|c|c|c|c|}
\hline \multicolumn{9}{|c|}{ Gesunde Hamster } \\
\hline Wurf Nr. & $\begin{array}{l}\text { Junge am } \\
\text { 1. 'Tg. }\end{array}$ & 7. Tg. & $\begin{array}{l}\text { Ausfall } \\
1 .-7 \mathrm{Tg} . \\
\%\end{array}$ & $\begin{array}{l}\text { Junge am } \\
\text { 14. Tg. }\end{array}$ & $\begin{array}{l}\text { Ausfall } \\
7 .-14 . \mathrm{Tg} . \\
\%\end{array}$ & $\begin{array}{l}\text { Junge am } \\
21 \text {. Tg. }\end{array}$ & $\begin{array}{l}\text { Ausfall } \\
14 .-21 . \mathrm{Tg} . \\
\%\end{array}$ & $\begin{array}{l}\text { Ausfall } \\
1 .-21 . \mathrm{Tg} . \\
\%\end{array}$ \\
\hline $\begin{array}{l}1 \\
3 \\
2 \\
4\end{array}$ & $\begin{array}{l}560 \\
447 \\
366 \\
225\end{array}$ & $\begin{array}{l}481 \\
418 \\
351 \\
218\end{array}$ & $\begin{array}{r}14.4 \\
3.4 \\
3.1 \\
3.1\end{array}$ & $\begin{array}{l}470 \\
414 \\
348 \\
209\end{array}$ & $\begin{array}{l}2.3 \\
1.0 \\
0.9 \\
4.1\end{array}$ & $\begin{array}{l}469 \\
387 \\
348 \\
208\end{array}$ & $\begin{array}{l}0.2 \\
6.5 \\
- \\
0.5\end{array}$ & $\begin{array}{r}16.3 \\
13.4 \\
4.9 \\
7.6\end{array}$ \\
\hline $1-4$ & 1598 & 1468 & 8.1 & 1441 & 1.8 & 1412 & 2.1 & 11.6 \\
\hline \multicolumn{9}{|c|}{ Diabetische Hamster } \\
\hline 1 & 396 & 218 & 44.9 & 205 & 6.0 & 203 & 1.0 & 48.7 \\
\hline
\end{tabular}

Tabelle 4. Ketonurie bei diabetischen Hamstern

\begin{tabular}{|c|c|c|c|c|c|c|c|c|c|}
\hline \multirow[t]{2}{*}{ Generation } & \multicolumn{3}{|c|}{ männliche Tiere } & \multicolumn{3}{|c|}{ weibliche Tiere } & \multicolumn{3}{|c|}{ getestete Hamster insgesamt } \\
\hline & $\begin{array}{l}\text { getestet } \\
n\end{array}$ & positiv & $\begin{array}{l}\text { positiv } \\
\% \\
\end{array}$ & $\begin{array}{l}\text { getestet } \\
n\end{array}$ & $\begin{array}{l}\text { positiv } \\
n\end{array}$ & $\begin{array}{l}\text { positiv } \\
\%\end{array}$ & $\begin{array}{l}\text { getestet } \\
n\end{array}$ & $\begin{array}{l}\text { positiv } \\
n\end{array}$ & $\begin{array}{l}\text { positiv } \\
\%\end{array}$ \\
\hline $\begin{array}{ll}F^{7} & 3 \\
F^{7} & 4 \\
F^{2} & 5 \\
F & 6 \\
F^{2} & 7\end{array}$ & $\begin{array}{r}4 \\
61 \\
104 \\
89 \\
2\end{array}$ & $\begin{array}{r}1 \\
53 \\
85 \\
73 \\
2\end{array}$ & $\begin{array}{r}25.0 \\
86.9 \\
81.7 \\
82.0 \\
100.0\end{array}$ & $\begin{array}{r}3 \\
72 \\
110 \\
98 \\
8\end{array}$ & $\begin{array}{r}3 \\
49 \\
66 \\
71 \\
7\end{array}$ & $\begin{array}{r}100.0 \\
68.1 \\
60.0 \\
72.4 \\
87.5\end{array}$ & $\begin{array}{r}7 \\
133 \\
214 \\
187 \\
10\end{array}$ & $\begin{array}{r}4 \\
102 \\
151 \\
144 \\
9\end{array}$ & $\begin{array}{l}36.4 \\
43.4 \\
41.4 \\
43.5 \\
47.7\end{array}$ \\
\hline$F 3-F 7$ & 260 & 214 & 82.3 & 291 & 196 & 67.35 & 551 & 410 & 74.4 \\
\hline
\end{tabular}

Tabelle 5. Auftreten von Diabetes mellitus in der Parental- und der 1.-7. Filialgeneration

\begin{tabular}{|c|c|c|c|c|c|c|c|c|c|}
\hline \multirow[t]{2}{*}{ Generation } & \multicolumn{3}{|c|}{ männliche Tiere } & \multicolumn{3}{|c|}{ weibliche Tiere } & \multicolumn{3}{|c|}{ Hamster insgesamt } \\
\hline & $\begin{array}{l}\text { getestet } \\
n\end{array}$ & $\begin{array}{l}\text { diabetisch } \\
n\end{array}$ & $\begin{array}{l}\text { diabetisch } \\
\%\end{array}$ & $\begin{array}{l}\text { getestet } \\
n\end{array}$ & $\begin{array}{l}\text { diabotisch } \\
n\end{array}$ & $\begin{array}{l}\text { diabetisch } \\
\%\end{array}$ & $\begin{array}{l}\text { getestet } \\
n\end{array}$ & $\begin{array}{l}\text { diabetisch } \\
n\end{array}$ & $\begin{array}{l}\text { diabetisch } \\
\%\end{array}$ \\
\hline$P$ & 5 & 1 & 20.0 & 8 & 1 & 12.5 & 13 & 2 & 15.4 \\
\hline$F^{1}$ & 7 & - & - & 17 & 1 & 5.9 & 24 & $\overline{1}$ & 4.2 \\
\hline$F 2$ & 70 & 20 & 28.6 & 61 & 13 & 21.3 & 131 & 33 & 25.2 \\
\hline$F 3$ & 128 & 33 & 25.8 & 118 & 37 & 31.4 & 246 & 70 & 28.5 \\
\hline$F^{4} 4$ & 648 & 135 & 20.8 & 558 & 171 & 30.6 & 1206 & 306 & 25.4 \\
\hline$F 5$ & 1061 & 183 & 17.2 & 1015 & 278 & 27.4 & 2076 & 461 & 22.2 \\
\hline$F 6$ & 879 & 124 & 14.1 & 758 & 168 & 22.2 & 1637 & 292 & 17.8 \\
\hline$F 7$ & 19 & 2 & 10.5 & 26 & 7 & 26.9 & 45 & 9 & 20.0 \\
\hline$P-F 7$ & 2817 & 498 & 17.7 & 2561 & 676 & 26.4 & 5378 & 1174 & 21.8 \\
\hline
\end{tabular}


leistung (Tucker 1966), die gesteigerte Milchproduktion reicht aber bei großen Würfen nicht zur optimalen Aufzucht der Jungtiere aus. Die höhere absolute Zuchtleistung bedingt also ein verringertes Körpergewicht des einzelnen Jungen.

Das von uns beobachtete Geburtsgewicht von 1.59 bis $1.94 \mathrm{~g}$ und das Absetzgewicht am 21. Tag von 13.65 bis $16.07 \mathrm{~g}$ wurde auch von Moore (1965) und von Herter und Rauch (1956) mitgeteilt.

Die praktische Bedeutung der gleichmäBigen Gewichtsentwicklung von Streifenhamstern aus Würfen mit verschiedener Anzahl Junger würde darin bestehen, daß für Experimente gleichalte Tiere mit gleichem Gewicht zur Verfügung stünden.

Der Vergleich erster Würfe diabetischer mit ersten Würfen nichtdiabetischer Hamster ergibt, daß signifikante Gewichtsunterschiede nur zwischen Nachkommen aus Würfen mit 5 Jungen am 14. und 21. Lebenstag bestehen. Für alle anderen Wurfgrößen konnten keine Unterschiede gefunden werden. Dies und der nur sehr geringe zahlenmäßige Unterschied (1.16 g nach 14 Tagen bzw. $1.88 \mathrm{~g}$ nach 21 Tagen) zeigt aber, daß der statistisch gesicherte Unterschied biologisch bedeutungslos ist.

Die Schädigungen der Jungen durch die diabetische Stoffwechsellage des Muttertieres kommt durch die hohe Mortalitätsrate zum Ausdruck. Sie führen entweder zum Exitus der Jungen, der vorwiegend innerhalb der ersten 7 Lebenstage auftritt oder nicht zu einer Beeinträchtigung der Entwicklungsfähigkeit der Jungen bis zum 21. Lebenstag, soweit sich diese am Körpergewicht ablesen läßt.

Die diabetische Stoffwechsellage des Muttertieres bedingt außerdem das gehäufte Vorkommen kleiner Würfe. Die geringere Anzahl von Implantaten am 8. Tag der Trächtigkeit bei diabetischen gegenüber normalen Hamstern spricht dafür, daß die Fertilitätsstörungen bei diabetischen Hamstern bereits vor der Nidation auftreten.

Die Mortalitätsrate von $\mathbf{1 1 . 6 \%}$ bei Jungen nichtdiabetischer Muttertiere und von $48.7 \%$ bei Jungen diabetischer Hamster stimmt mit den Ergebnissen von Butler (1967) überein.

Für die Tatsache, daß sich der Diabetes in höheren Inzuchtgenerationen früher manifestiert, haben wir keine Erklärung.

Das von Moore (1965) erwähnte Zuchtsystem, bei dem Wurfgeschwister beisammenbleiben und nur tragende Weibchen separiert und nach dem Absetzen der Jungen am 21. Tag post partum wieder zu den Geschwistern gesetzt werden, hat sich bewährt.

Die von anderen Autoren (Yerganian 1958 und Moore 1965) für eine erfolgreiche Zucht des Chinesischen Streifenhamsters geforderte Notwendigkeit einer Dunkelperiode von konstant 11 Stunden ist nach unseren Erfahrungen nicht notwendig. Die Tiere vermehren sich auch dann gut, wenn Tageslicht zu den Zuchträumen Zugang hat, wodurch jahreszeitliche und wetterbedingte Schwankungen der Beleuchtungsdauer und -intensität auftreten.

Abschließend möchten wir feststellen, daß wir uns durchaus darüber im klaren sind, daß die geringe Anzahl von 361 Nachkommen aus 83 Würfen diabetischer Mütter nur sehr vorsichtige Aussagen zuläßt.

Für die Hilfe bei der Erfassung und Auswertung der Ergebnisse möchten wir Frau Michalik und für die statistische Bearbeitung des Zahlenmaterials Herrn Dr. Hartmann unseren Dank aussprechen.

\section{Literatur}

1. Butler, L.: The Inheritance of Diabetes in the Chinese Hamster. Diabetologia 3, 124-129 (1967).

2. Gerritsen, G.C., Dulin, W.E.: Characterization of Diabetes in the Chinese Hamster. Diabetologia 3, 7484 (1967).

3. Hammond, J.: The fertilization of rabbit ova in relation time. A method for controlling the duration of pregnancy and the weight of the young at birth. J. exp. Biol. 11, 140-151 (1934).

4. Herter, K., Rauch, H.-G.: Haltung und Aufzucht Chinesischer Zwerghamster. Z. f. Säugetierkunde 21, $161-166$ (1956).

5. Ibsen, H.L.: Prenatal growth in guinea-pigs with special reference to environmental factors affecting weight at birth. J. exp. Biol. 51, $51-57$ (1928).

6. King, H.D.: Birth weight in the grey Norway rat and the factors which influence it. Anat. Rec. 63, 335-342 (1935).

7. Meier, H.: Spontaneous diabetes mellitus in the Chinese hamster (Cricetulus griseus). II. Findings in the offspring of diabetic parents. Diabetes 10, 12-18 (1961).

8. - Yerganian, G.A.: Spontaneous hereditary diabetes mellitus in Chinese hamsters (Cricetulus griseus). I. Pathological findings. Proc. Soc. exp. Biol. (N.Y.) 100, $810-816(1959)$.

9. - - Spontaneous hereditary diabetes mellitus in the Chinese hamster (Cricetulus griseus). IIr. Maintenance of a diabetic hamster colony aided by the hypoglycemic therapy. Diabetes 10, 19-21 (1961).

10. Moore, W.: Observations on the breeding and care of the Chinese hamster, Cricetulus griseus. Lab. Anim. Care 15, 94-101 (1965).

11. Omtvedt, I.T., Whatley, J.A., Willham, R.L. : Some production factors associated with weaning records in swine. J. Anim. Sci. 25, 372-376 (1966).

12. Opicha, L., Opicha, M.: Die Bedeutung der Wurfgröße für das Wachstum der Ferkel bis zum Alter von 4 bzw. 8 Wochen. Z. f. Tierzucht u. Z. f. Biol. 56, 135-141 (1944).

13. Smith, C.: The introduction and breeding of the Chinese striped hamster (Cricetulus griseus) in Great Britain. J. Anim. Techns. Ass. 7, 59-60 (1957).

14. Smith, A.D., Donald, H.P.: Weaning weight of pigs and litter sampling with reference to litter size. J. Agr. Sci. 27, 485-489 (1937).

15. Steinbeck, H., Berswordt-Wallrabe, R. von: Leistungskontrolle einer konventionellen Rattenzucht. Z. f. Versuchstierkunde 8, 167-186 (1966).

16. Tucker, H.A.: Regulation of mammary nucleic acid content by various suckling intensities. Amer. $J$. Physiol. 210, 1209-1215 (1966).

17. Weber, E. : Grundriß der biologischen Statistik. Stuttgart: Gustav Fischer Verlag 1967. 
18. Wishart, J., Hammond, J.: A statistical analysis of the interrelation of the litter size and duration of prenancy on the birth weight of rabbits. J. Agr. Sci. 23, $463-472$ (1933).

19. Yerganian, G.: The Striped-Back or Chinese Hamster, Cricetulus griseus. J. nat. Cancer Inst. 20, 705-721 (1958).

20. - The Chinese hamster (Cricetulus griseus). The UFAW handbook on the care and management of laboratory animals. 2nd Ed., Chap. 21, p. 340. Edinburgh and London: E. u. S. Livingstone Ltd. 1966.

Dr. O. Loge

Experimentelle Med. Forschung

Hauptlaboratorium der Schering AG.

D-1 Berlin 65

Müllerstr. $170-172$ 